\title{
Psicología de la salud: \\ Una clave para comprender el fenómeno de la adherencia terapéutica
}

\author{
Manuel O rtiz Pla, Eugenia O rtiz P2. \\ Health psychology: A key \\ to understand therapeutic adherence
}

The lack of compliance with treatment is a public health problem that affects the patient's quality of life, avoids an objective assessment of therapeutic effectiveness and may even cause death. Non compliance can be considered an onedimensional problem that only depends of patients's responsibility. However, Health Psychology has generated theoretical models that allow the prediction and understanding of therapeutic compliance. It also expands the responsibility to other agents involved in the therapeutic process, specifically health care providers and health systems (Rev Méd Chile 2007; 135: 647-52).

(Key words: Behavioral medicine; Compliance; Psychology, medical)

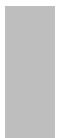

Recibido el 6 de abril, 2006. Aceptado el 17 de julio, 2006.

${ }^{1}$ Facultad de Psicología. Universidad Mayor, Temuco. ${ }^{2}$ Facultad de Medicina, Universidad del Desarrolo - Clínica Alemana.

apsicólogo, Magíster en Psicología

$\mathrm{L}$ a irrupción de nuevas tecnologías y tratamientos farmacológicos han posibilitado contar cada día con mejores y más variadas prescripciones médicas. Esto, a su vez, debiera significar un aumento tanto en la efectividad de los tratamientos, así como también incidir directamente en la calidad de vida de los pacientes. Sin embargo, para que esto ocurra debieran existir al menos dos condiciones: una adecuada prescripción por parte del equipo médico y un correcto cumplimiento de la misma. Debido a que la prescripción médica es un tema que corresponde sólo a competencias de médicos, esta revisión se centrará en la adherencia

Correspondencia a: Manuel Ortiz P. Universidad de La Frontera, Departamento de Psicología. Av. Francisco Salazar 01145, Temuco. Fono: (45)325605. Fax: (45)341480.

E mail: msortiz@uc.cl a los tratamientos médicos, aspecto conductual en el cual la psicología de la salud tiene mucho que aportar.

\section{LA IMPORTANCIA DE ESTUDIAR LA ADHERENCIA TERAPÉUTICA}

Varios son los motivos por los cuales estudiar la adherencia a los tratamientos. En primer lugar, gran parte de las indicaciones terapéuticas no pueden ser supervisadas cotidianamente por una agente externo al paciente $\mathrm{y}$, al contrario, quedan bajo su directa responsabilidad, entonces, es el paciente quien en definitiva decide si cumplir o no. En efecto, las bajas tasas de adherencia comunicadas, parecieran apoyar la idea de que el paciente mayoritariamente no cumple con el tratamiento. Por ejemplo, el National Heart, Lung 
and Blood Institute ${ }^{1}$ señala que entre $30 \%$ y $70 \%$ de los pacientes no cumple con los consejos entregados por sus médicos. Martín y $\mathrm{Grau}^{2}$ informan que, en general, $40 \%$ de los pacientes no cumplen las recomendaciones terapéuticas; en el caso de las enfermedades agudas este porcentaje llega a 20\%, mientras que en enfermedades crónicas el inclumplimiento alcanzaría a 45\%. Cuando los tratamientos generan cambios en los hábitos de vida e invaden diversas áreas (laboral, social, familiar), los porcentajes de no adherencia aumentan significativamente ${ }^{3}$. Por ejemplo, para el tratamiento de la diabetes mellitus tipo 1, se han encontrado tasas de no adherencia cercanas a $50 \% 4,5$ y además, se ha informado una relativa independencia entre los elementos pilares de la terapia, lo que significa que en parte los adolescentes diabéticos tipo 1 tienden a adherir más o menos bien a la dieta, al ejercicio o a la insulinoterapia $^{6}$, sin que el cumplimiento de uno implique el de los otros.

Por otra parte, si un paciente no cumple con las indicaciones médicas es imposible estimar el efecto del tratamiento, lo que a su vez puede contribuir a prolongar la terapia y perpetuar enfermedades o problemas de salud e incluso conducir a la muerte, con el consecuente costo económico para el estado, tanto en materias relativas a pérdida de productividad y costos asociados a rehabilitación y pensiones de invalidez (por ejemplo, el costo asociado al tratamiento de la hipertensión y sus complicaciones representó $12,6 \%$ del gasto total de atención de salud en Estados Unidos de Norteamérica en 1998) ${ }^{7}$.

\section{FACTORES QUE DETERMINAN LA NO ADHERENCIA TERAPÉUTICA}

Varios son los factores que se relacionan con este problema. Existen factores personales, del sistema o equipo de asistencia sanitaria y de la enfermedad y del tratamiento. Dentro de los factores personales, múltiples variables pueden mencionarse: falta de motivación, desconocimiento, baja autoestima, etc ${ }^{8}$. La depresión frecuentemente ha sido asociada con los resultados que se alcanzan en las enfermedades crónicas 9 . En relación a la DM1, al parecer, cuando la depresión está presente, existe evidencia de un peor control metabóli- co, disminución de la actividad física, mayor obesidad, y potencialmente mayores complicaciones derivadas de la patología ${ }^{10}$.

Sobre los factores del sistema de salud y los prestadores, pocos estudios se han realizado, no obstante esto, bien documentada está la relación entre adherencia y satisfacción con el equipo médi$\mathrm{co}$, al igual que el rol que desempeña la relación médico paciente. Cada día se ha ido estableciendo que una relación de colaboración entre el paciente y el o los prestadores, puede contribuir a mejorar la adherencia al tratamiento en enfermedades crónicas $^{11}$, además esta variable parecería asociarse con mejoras en la participación del paciente en su cuidado, con disminución de cancelaciones a las visitas médicas e incrementos en el compromiso del paciente con su tratamiento. A su vez, se ha demostrado que la satisfacción ${ }^{12}$ y la comunicación del paciente con el equipo de atención en salud, son variables que pueden contribuir a la adherencia al tratamiento en pacientes crónicos ${ }^{3}$.

Respecto a la enfermedad y su tratamiento, y tal como ya se ha mencionado, las tasas de adherencia parecen ser diferentes si se trata de una patología aguda versus una crónica ${ }^{2}$. Del mismo modo, cuando el tratamiento de la enfermedad consiste en un patrón complejo de conductas e invade diferentes aspectos del paciente o sus resultados son invalidantes, tiende a afectar la adherencia terapéutica. Se podría establecer la siguiente relación: a mayor complejidad del tratamiento, menos adherencia terapéutica.

ROL DE LA PSICOLOGÍA DE LA SALUD

EN EL FENÓMENO DE LA ADHERENCIA TERAPÉUTICA

Pese a que el fenómeno de la adherencia terapéutica involucra a todos los profesionales de la salud, la psicología de la salud proporciona un cuerpo de teorías y modelos conceptuales que han demostrado ser útiles para el pronóstico e intervención sobre la conducta de adherencia. A saber, la Teoría Social Cognitiva ${ }^{13}$, la Teoría de la Acción Razonada ${ }^{14}$, el Modelo de Creencias en Salud $^{15}$, el Modelo Transteórico ${ }^{16}$ y el Modelo de Información-Motivación-Habilidades Conductuales ${ }^{17}$. A continuación se presenta una breve descripción de cada una. 
Teoría Social Cognitiva. Sugiere que cualquier cambio de conducta (por ejemplo, volverse adherente) se fundamenta sobre la creencia de que una persona puede alcanzar exitosamente la conducta deseada. Esta creencia en la habilidad o capacidad de alcanzar lo deseado se denomina autoeficacia percibida. De acuerdo a la teoría, esta creencia es esencial para predecir la conducta de adherencia, aun cuando otros predictores están presentes. Es así como una persona puede sentirse vulnerable ante una enfermedad, entender y conocer cuáles son los comportamientos específicos que requiere el tratamiento, creer que la adopción de tal conducta saludable disminuirá la probabilidad de enfermar y tener apoyo social. Sin embargo, si la persona no está convencida que tiene la habilidad para ejecutar la conducta, es poco probable que la lleve a cabo. Esta teoría ha sido probada con pacientes diabéticos, informándose que para afrontar efectivamente las demandas conductuales impuestas por el tratamiento, se requiere un buen sentido de autoeficacia ${ }^{18}$. Asimismo, un estudio efectuado por Anderson ${ }^{19}$, indica que la autoeficacia es una variable que puede conducir a mejorar el control de las glicemias de los pacientes. Además, señala que esta variable correlaciona positivamente con las conductas de salud deseadas en el tratamiento de la diabetes. Finalmente, se informa que la variable autoeficacia, incrementa la habilidad del paciente para guiar su tratamiento, de modo independiente al equipo médico, lo que a su vez podría conllevar menos visitas a los centros de salud.

Teoría de la Acción Razonada. Relaciona las creencias, actitudes, intenciones y conducta. Señala específicamente que el mejor predictor de la conducta es la intención de llevarla a cabo. A su vez, la intención es predicha por la actitud hacia ejecutar tal conducta y factores sociales, tales como la percepción que los sujetos significativos para el paciente tienen acerca de tal conducta. Tomando en consideración lo sugerido por la teoría, se podría predecir con mayor exactitud que una persona se involucre en conductas saludables indagando sobre la intención que tenga de realizarlas.

Modelo de Creencias en Salud. Integra teorías cognitivas y conductuales para explicar porqué la gente falla en adherir a conductas saludables, considerando el impacto de las consecuencias y expectativas relacionadas con la conducta. Específicamente, la probabilidad que una persona adopte o mantenga una conducta para prevenir $\mathrm{O}$ controlar una enfermedad dependerá de: (a) grado de motivación e información que tenga sobre su salud, (b) autopercepción como potencialmente vulnerable ante la enfermedad, (c) percibir la condición como amenazante, (d) estar convencido que la intervención o tratamiento es eficaz y (e) percepción de poca dificultad en la puesta en marcha de la conducta de salud. Apoyo empírico a esta teoría ha sido reportado por varios autores. McCord y Brandenburg ${ }^{20}$, informaron que aquellos diabéticos que toman en serio su enfermedad, tienen más probabilidad de adherir a su tratamiento. También se ha señalado que aquellos pacientes no adherentes, creen que la enfermedad no afectará sus vidas. Estos pacientes presentaron al mismo tiempo menor intención de cumplir las instrucciones del médico. Contrariamente, una fuerte creencia en la medicina convencional aumentaría la probabilidad de adherir ${ }^{21}$.

Modelo transteórico. Propone etapas de cambio para explicar la adquisición de conductas saludables o la reducción de conductas de riesgo. Se distingue cinco etapas diferentes para el cambio: 1) Precontemplación (el individuo no tiene intención para cambiar en el corto plazo, usualmente medido en los próximos 6 meses); 2) Contemplación (el sujeto no está preparado para tomar acciones en el presente, pero podría intentarlo en el corto plazo de aquí a 6 meses); 3) Preparación (el individuo está considerando activamente cambiar su conducta en el futuro inmediato, dentro del próximo mes); 4) Acción (el sujeto ha hecho un cambio en el pasado reciente, pero este cambio no está bien establecido); 5) Mantenimiento (se ha cambiado la conducta por más de 6 meses y se está activamente involucrado para sostenerla $^{22}$. De acuerdo a este modelo es posible predecir que los pacientes con alguna enfermedad crónica que se encuentren en las etapas de acción (acción y mantenimiento) en contraposición de aquellos que se encuentren en las etapas de preacción (precontemplación, contemplación y preparación) ingieran menos alimentos hipercalóricos, coman durante el día más frutas y vegetales, 
hayan tenido más visitas al médico, así como también hayan fumado menos, presenten menos problemas psicológicos, etc ${ }^{23}$.

\section{Modelo de Información-Motivación-Habilidades} Conductuales. Sometido a rigurosa investigación empírica, tanto en estudios prospectivos como correlacionales, este modelo demuestra que en conjunto la información, la motivación y las habilidades conductuales explican 33\% de la varianza del cambio conductual. Específicamente, demuestra que la información es un prerrequisito pero por sí sola no es suficiente para alterar la conducta. Además provee evidencia que la motivación y las habilidades conductuales son determinantes críticos que son independientes del cambio conductual. La información y la motivación afectarían la conducta por medio de las habilidades conductuales. Sin embargo, cuando las habilidades conductuales son familiares o no complicadas, la información y la motivación pueden actuar directamente sobre la conducta. En este caso, un paciente puede seguir una prescripción basado en la información entregada por el médico. En el modelo, la relación entre motivación e información es débil. Sin embargo, la presencia de ambos aumenta el poder predictivo del modelo.

Desafíos Que IMPONE EL FENÓMENO DE LA ADHERENCIA TERAPÉUTICA A LOS PROFESIONALES DEL ÁREA DE LA SALUD

Por tratarse de un problema de salud pública que incluye múltiples factores y que además posee una naturaleza compleja donde los factores comportamentales y los subjetivos desempeñan un rol importante, se considera a la adherencia a los tratamientos un problema aún no resuelto. En este sentido, los desafíos que impone el fenómeno de la adherencia terapéutica podrían agruparse en las siguientes áreas: definición del concepto de adherencia, evaluación o medición de la adherencia terapéutica e intervención para incrementarla, todas las cuales se encuentran íntimamente relacionadas.

Cómo se debe entender la adherencia terapéutica. Actualmente no existe acuerdo aún sobre una definición unívoca del concepto. Es frecuente encontrarse en la literatura con conceptos tales como cumplimiento, adherencia, cooperación, colaboración, obediencia, observancia, alianza terapéutica, seguimiento, adhesión y concordancia $^{24}$. Sin embargo, una definición bien difundida es la de Sackett y Haynes ${ }^{25}$, quienes conceptualizan la adherencia como una situación en que la conducta del paciente se corresponde con las instrucciones clínicas entregadas. Haynes ${ }^{26}$ define adherencia como la magnitud en cual la conducta de una persona coincide con las recomendaciones hechas por el equipo de salud. Detractores de esta definición, argumentan que otorga un rol paternalista y coercitivo al equipo médico, limitando la posibilidad que el paciente asuma un rol más relevante. En este contexto, Hentinen y Kyngäs ${ }^{27}$ definen el concepto como un proceso activo, intencional y responsable de cuidado, en el cual el sujeto trabaja para mantener su salud en estrecha colaboración con el personal de salud. Una de las definiciones más aceptadas es la propuesta por Di Mateo y Di Nicola ${ }^{28}$, quienes conceptualizan la adherencia como una implicación activa y de colaboración voluntaria del paciente en un curso de comportamiento aceptado de mutuo acuerdo, con el fin de producir un resultado preventivo o terapéutico deseado. Esta definición tiene la virtud de otorgar una mayor participación del paciente en la toma de decisiones que afectan a su propia salud. En este caso el paciente debe adherirse a un plan que acepta y ha acordado con el médico tratante y en el cual se supone ha podido participar en su elaboración.

Ahora bien, la dificultad de consensuar una definición única de adherencia hace difícil su operacionalización y medición. Como un concepto conductual, la adherencia implica acciones complejas, emociones y fenómenos que pueden no ser directamente observables ${ }^{29}$. Los métodos directos para evaluar adherencia, tales como los exámenes de sangre y de orina son cuestionados pues miden resultados más que el proceso de adherencia. De este modo, métodos indirectos, como las entrevistas, son generalmente utilizados, debido a que poseen la ventaja de presentar la propia evaluación del paciente respecto de su adherencia. La observación de la conducta del paciente no es usualmente relevante, debido a que es un proceso muy complicado e incluso resulta imposible seguir al paciente durante todo 
el día ${ }^{30}$. La evaluación realizada por enfermeras y médicos ha sido usualmente realizada sobre la base de los resultados obtenidos a través de exámenes de sangre u orina o de entrevistas. Sin embargo, resulta ser poco confiable en la evaluación de la adherencia, debido a que no considera la conducta del paciente ${ }^{30}$. Todo indicaría que al parecer sería interesante desarrollar mecanismos o instrumentos que tiendan a considerar la integración de métodos directos e indirectos para evaluar

\section{REFERENCIAS}

1. National Heart, Lung And Blood Institute. Behavioral research in cardiovascular, lung, and blood health and disease. Washington, DC: U.S. Department of Health and Human Services, 1998.

2. Martin L, Grau J. La adherencia terapéutica como un problema de la psicología de la salud. En: Hernández E, Grau J, eds. Psicología de la Salud. Fundamentos y Aplicaciones. Universidad de Guadalajara, 2005.

3. Rodríguez M. Psicología Social de la Salud. Madrid: Pirámide, 2001.

4. Ortiz M, Ortiz E. Adherencia al tratamiento en adolescentes diabéticos tipo 1 chilenos: Una aproximación psicológica. Rev Méd Chile 2005; 133: 307-13.

5. Codner E, Merice V, Román R, Hric i, Martínez A, Unanue $\mathrm{N}$ et al. Nuevos esquemas de tratamiento con insulina en niños y adolescentes con Diabetes Mellitus tipo 1 (DM1) en un Hospital Público. Rev Chil Pediatr 2004; 75: 520-9.

6. Weissberg-Benchell J, Glasgow aM, tinan WD. Adolescent diabetes management and mismanagement. Diabetes Care 1995; 18: 77-82.

7. Hodgson TA, Car L. Medical care expenditures for hypertension, its complications, and its comorbidities. Medical Care 2001; 33: 599-615.

8. ORTIZ M. Factores psicológicos y sociales asociados a la adherencia al tratamiento en adolescentes diabéticos tipo 1. Psykhe 2004; 13: 21-31.

9. Goldney R, Phillips P, Fisher L, Wilson D. Diabetes, depression and quality of life. Diabetes Care 2004; 27: 1066-70.

10. Lin E, Katon W, Von Korff M, Rutter C, Simon G, Oliver M et AL. Relationship of Depression and Diabetes Self-Care, Medication Adherence, and Preventive Care. Diabetes Care 2004; 27: 2154-60. la adherencia terapéutica, y por el momento complementar ambos métodos.

Finalmente, sobran argumentos para efectuar intervenciones que contribuyan a la adherencia terapéutica y se justifican por sí mismos. Sin embargo, parece necesario reflexionar que por tratarse de un fenómeno multidimensional, las intervenciones deben dirigirse a todos los estamentos involucrados, vale decir, pacientes, prestadores de salud y sistemas de salud.

11. Ciechanowski P, Katon W, Russo J, Walter E. The patient - provider relationship: Attachment theory in diabetes. Am J Psychiatr 2001; 158: 29-35.

12. Von Korff M, Gruman J, Schaefer J, Curry S, Vagner E. Collaborative management of chronic illness. An Inter Med 1997; 127: 1097-102.

13. Bandura A. Self-efficacy mechanism in human agency. Am Psychol 1982; 37: 122-47.

14. AJZEN I, Fishibein M. Undestanding attitudes and predicting social behavior. Englewood Cliffs, NJ: Prentice Hall, 1980.

15. Rosentock I. Historical origins of the health belief model. Health Educ Monogr 1974; 2: 1-8.

16. Prochaska J, Di Clemente C. Transtheoretical therapy: Toward a more integrative model of change. Psychother Theory Res Pract 1982; 19: 276-88.

17. Fisher J, Fisher W, Miscovich S, Kimble D, Malloy T. Changing AIDS risk behaviour: Effects of an intervention emphasizing AIDS risk reduction information, motivation, and behavioural skills in a college student population. Health Psychol 1996; 15: 114-23.

18. Van Der Ven N, Weinger K, Yi J, Pouwer F. The confidence in diabetes self-care scale: Psychometric properties of a new measure of diabetes-specific self-efficacy in Dutch and U.S. patients with type 1 diabetes. Diabetes Care 2003; 26: 713-9.

19. Anderson R. The diabetes empowerment scale: A measure of psychosocial self - efficacy. Diabetes Care 2000; 11: 519-26.

20. McCord E, Brandenburg C. Beliefs and attitudes of persons with diabetes. Family Medicine 1995; 27: 276-1.

21. Garay-Sevilla M, Malacara H, González-Parada F, JORDAN-GINES L. The belief in convencional medicine and adherence to treatment in non-insulindependent diabetes mellitus patients. J Diabetes Complications 1998; 12: 239-45. 
22. Ruggiero L, Prochaska J. Readiness for change: applications of the transtheoretical model to diabetes. Diabetes Spectrum 1993; 6: 22-60.

23. VAluIs M. Stages of change for healthy eating in diabetes. Diabetes Care 2005; 26: 1468-74.

24. Basterra M. El cumplimiento terapéutico. Pharmaceutical Care 1999; 1: 97-106.

25. SaCkett DL, Haynes B. Compliance with Therapeutic Regimens. Baltimore: Johns Hopkins University Press, 1976.

26. HAYNES RB. Strategies to improve compliance with referals, appointments, and prescribed medical regimens. En: Haynes RB, Taylor DW, Sackett DL. eds. Compliance with health care. Baltimore: J. Hopkins University Press, 1979.

27. Hentinen M, Kyngäs H. Compliance of young diabetic's with health regimens. J Adv Nurs 1992; 17: 530-6.

28. Di Matteo R, Di Nicola D. Achieving Patient Compliance. The Psychology of the Medical Practitioner's Role. New York N.Y: Pergamon, 1985.

29. Morse JM, Mitcham C, Hupsey JE, Tason MC. Criteria for concept evaluation. J Adv Nurs 1996; 24: 385-90.

30. KYNGäs H. Compliance of adolescents with diabetes. Int Pediatr Nurs 2000; 15: 260-7. 